\title{
Discussion on Design Methods for Protection and Renovation of Traditional Dwellings in North Shandong
}

\author{
Yanru Gai \\ Binzhou University, Binzhou, Shandong, 256600, China
}

\begin{abstract}
Traditional dwellings in north Shandong act as an integral part of the Culture of Yellow River. Its value is being recognized by the society gradually. Many protection theories of historical buildings, architectural aesthetics, many cases of building renovation and protection have been proposed. In this paper, we explore and discuss the design methods in renovation of dwellings in north Shandong to find a new road for new rural areas in north Shandong, with remaining the architectural style and cultural tradition of traditional dwelling.
\end{abstract}

Keywords: dwellings in North Shandong, building renovation, building protection

Published Date: March 2018

Published Online: $31^{\text {st }}$ March 2018

\section{Corresponding Author:}

Yanru Gai, 148087739@qq.com

\section{Introduction}

Shandong province has a varied topography, with north, central and south Shandong together with peninsula and coastal areas being dominated by alluvial plain of Yellow River, mountains and hills, respectively. Different topographies and geologies, natural conditions, social and cultural factors and other factors contribute to different styles and features of dwellings in Shandong in aspects of structural style, planar characteristics, external characteristics and other aspects.

Thus far, most studies have focused on quadrangle dwellings in Jinan, seaweed dwellings in coastal areas of Jiaodong Peninsula, stone buildings in central mountainous areas and manor buildings in Qixia in Shandong province. However, while less study has been made on traditional dwellings in coastal areas of Yellow River in north Shandong. By 2016, 6,712 traditional villages were incorporated in the Traditional Chinese Villages Catalog, only 37 of which reside in Shandong. Further, there is little research works on villages in north Shandong. The research works mainly include Dwellings in Shandong Province, Dwellings in Shandong Culture Series, Buildings and Architectural Culture of Shandong in The Series of Cultural Relics in Shandong. These books introduce the dwellings in Shandong spanning the perspectives of spatial form, art composition, folk customs and featured dwellings. Nonetheless, scarce papers and literature have been provided on dwellings in north Shandong. The Study on Regional Culture of Dwellings in Northwest Shandong compiled by Menghan Wang in 2014 proposed the traits of regional culture of traditional dwellings in northwest and north Shandong. Despite all these, publications concerning renovation and protection design methods of traditional dwelling in north Shandong are still absent. Given such a situation, this paper takes dwellings in north Shandong as the main research object to discuss the design methods for protection and renovation of such dwellings based on the current social demands.

However, the protection and renovation of traditional dwelling are plagued with two grave problems. First, the atmosphere of nostalgia has been prevailing. In an era with rapid economic development, many traditional villages are damaged in a ruthless manner in tourism development, where developers set asides the historical context of buildings to imitate and copy historical and cultural buildings or cluster relics, leading to damage of the building value and context. Second, in "complete and dyed-in-the-wool renovation", the authenticity of the architectural style of traditional dwellings and protection of physical relics are neglected. These behaviors "confuse the true and false of historical buildings and damage the buildings' cultural context. In addition to leading to misunderstanding of visitors, it also results in misinterpretation of the protection meaning", and decrease the historical value of traditional dwellings. ${ }^{[1]}$

In face of the above problems, we should hold a prudent and respectful attitude towards protection and renovation of traditional villages to prevent the reoccurrence such tragedies. Looking back on the history, theories about building protection, repair and renovation arise one after another and some of Volume2; Issue 2 33 
influential theories have been checked in practices and remain with strong vitality till now. For instance, the "authenticity" theory has been followed as the principle for protection and renovation of architectural heritage and other similar buildings since the introduction of The Athens Charter in 1931. Furthermore, the "reversibility" and "minimal intervention" theories have also exerted profound influence.

The three theories have a similar purpose, which is to renovate and protect historical buildings while not changing the traits of the original buildings. Therefore, design practices under the guidance of these principles can protect the regional cultural value and style and characteristics of historical buildings and play a positive propelling role in modern transition of historical buildings. These three principles have varied manifestation forms, design means and design methods in renovation and protection practices of general buildings. Among them, the most typical manifestation forms are the following:

\section{Maintain and restore the original appearance of buildings}

Such a mean follows the design principle of maintaining the original historical appearance and style of the building, which basically maintain the external appearance of buildings and transmit the internal structure, functions and structures (Figure 1). In the renovation, the external appearance of the building remains basically unchanged and the renovation and design of internal finishes and overall structure of buildings are taken as the focus.

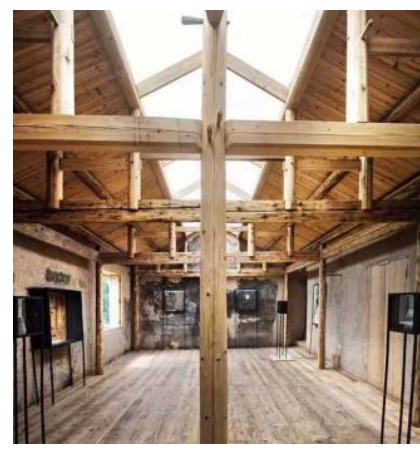

Figure 1. Dwelling in Songyang village

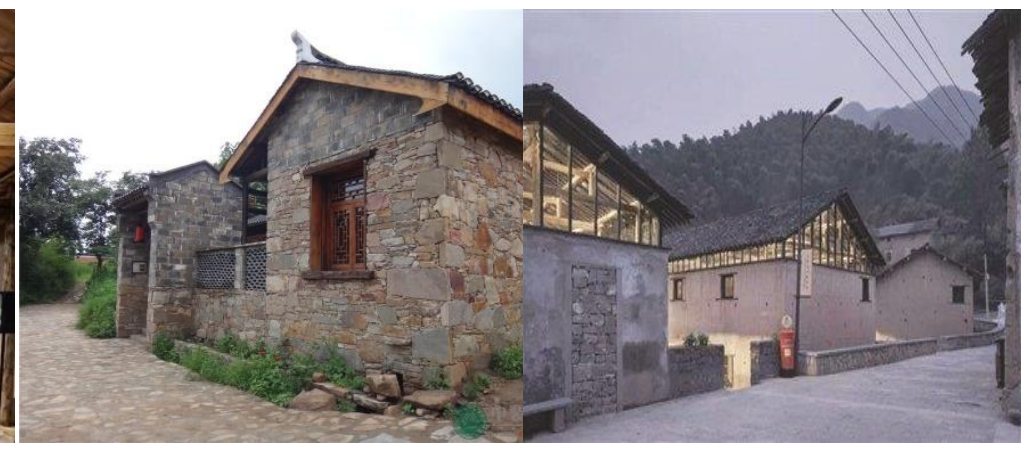

Figure 2. Renovation of Taoyuan village
Figure 3. Xianfeng Yunxi library

\section{Harmony of new and old elements}

The main external shape of the building remains unchanged and local parts of the buildings are added or decreased (Figure 2) to maintain the entire style and appearance of the building. There is no accurate restoration or duplication is made for damaged parts of the original building and no comparison design is made between new and old materials. The added part echoes to the original building in external appearance, achieving overall unity and integrity in the end.

\section{Design comparison of new and old elements}

This design method is used to distinguish from the original building's part and avoid confusion (Figure $3)$. The original part of the building can be distinguished clearly in observation and application.

To maintain the original architectural style and characteristics of dwellings in northwest Shandong, the typical style and characteristics of dwellings need to be extracted first to renovate and design dwellings by making use of the above three principles. In China, many traditional dwellings have their own unique architectural style and characteristics. For instance, quadrangle dwellings in Beijing, cave dwellings in Shanxi, stone houses in lanes in Shanghai, blockhouses in Tibet, Wannan houses and other traditional dwellings have unique styles and characteristics, which contain a large volume of historical and cultural information, including architectural pattern, style and details, people's lifestyle, local culture, tradition, religious belief and historical influence in certain special periods, etc.

After investigation and survey, the typical styles and characteristics of dwellings in north Shandong are mainly manifested in their courtyard form and building materials. Most dwellings in north Shandong are Volume2; Issue 2 
of three-section compound structure (Figure 6). Wherein, the North House mainly plays a functional role and uses for residence and living. Houses in the east and west are wing-rooms and mainly for storing, livestock raising and cooking. The principal room has a higher storey height, while wing-rooms on the east and west have a lower storey height and are simpler in construction, modeling and material selection. The main entrance is mainly located in the southeast corner of the courtyard. The axis of most courtyards is in south-to-north direction. The addition of new parts in most traditional dwellings or courtyards will change the original physical proportion of buildings and the overall form and historical styles and characteristics of buildings. To avoid these from happening, the following can be done:

\subsection{Strengthen the original axis}

To highlight the original architectural styles and characteristics of dwellings in north Shandong, the axis characteristics and courtyard's layout remain unchanged to strengthen the original axis. Several methods to strengthen the original axis has been proposed, which can be divided into two categories, including the spatial axis of the original buildings (chokkura Square in Janpan) and making new axis to contrast with the axis of the original buildings.

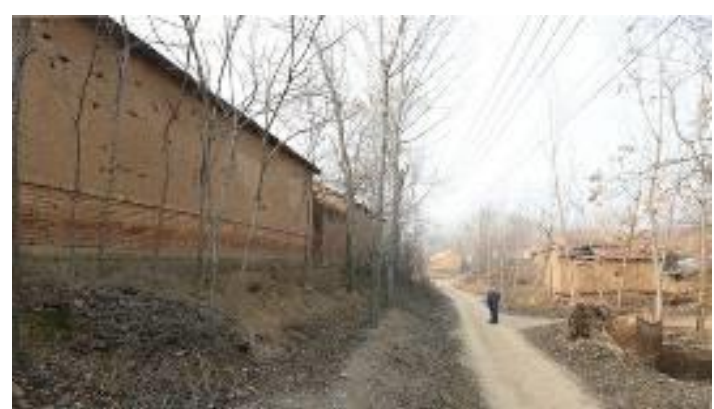

Figure 4. Main street of Xizhifang village the newly added building materials is to that in the original building environment, the closer the relationship between them. Moreover, its integrity is also highlighted (Figures 4 and 5). Texture design of materials include shape, void ratio, texture and gap of materials. The tactile texture includes pressure sensation, elasticity, plasticity, brittleness, tenacity, smoothness and roughness, abrasion resistance, etc. To avoid building's original texture from damage, the design principles of "material blanking, homogenization of material texture and quality homogenization of material" should be followed in material

\subsection{Integration and unity}

To make the newly added part in accordance with the environmental style of the original building, spatial three-dimensional extension can be made in many aspects to render a unified new image to the newly added part with the original building. Dwellings inevitably suffer some damages in long-term use and their architectural composition and space cannot meet the current use demands to a large extent. Moreover, adding new building blocks or components is unavoidable in renovation. The new building blocks or components should follow the principle of "integration and unity" and many design measures can be adopted in renovation, such as interspersing of new and old building blocks, juxtaposition on the right and left sides and juxtaposition up and down and concave-convex blocks.

Material design is also an integral part in the renovation of dwellings. Material design mainly comes from people's visual perception and tactile sense, which are dominated by these two feelings, the focus of material design is color, texture and quality design. The color design is to protect the original styles and characteristics of dwellings, thus should follow the principles of "similar dominant colors" and "harmonious color". ${ }^{[2]}$ The more similar or closer the color of

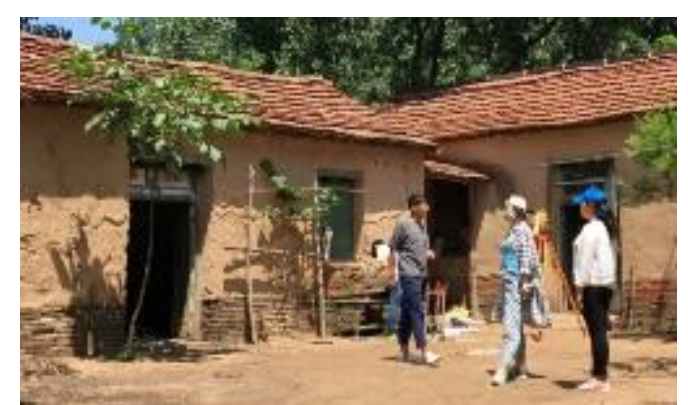

Figure 5. A residential courtyard in Xizhifang village

texture design. ${ }^{[2]}$

Given northwest Shandong resides over both banks of Yellow River and lack such building materials as timber and masonry, dwellings in north Shandong are built by inexhaustible loess. ${ }^{[3]}$ After adding water and wheat straw into loess and mixing evenly, the mixture is poured into the square die for shaping. Sun-dried mud brick represents typical building materials in dwellings in north Shandong. Its general proportion is 9:1, and it is about $40-42 \mathrm{~cm}$ long, about $30 \mathrm{~cm}$ wide and $8-10 \mathrm{~cm}$ high mortar joint is about $1 \mathrm{~cm}$. The surface of building walls is plastered Volume2; Issue 2 
by slurry and the bottom of most walls is constructed by sintered red bricks. Therefore, sun-dried mud walls of dwellings in north Shandong are mainly composed of sintered bricks, sun-dried mud bricks and slurry. ${ }^{[4]}$

Most dwellings have rather unclear stylistic characteristics in building appearance before renovation due to poor durability and protection of building materials. "Subtraction operation" can be done to eliminate parts hindering the dwellings' architectural style and the most valuable part should be maintained before renovation and protection. ${ }^{[5]}$ The external walls of the building are constructed by sintered red bricks as depicted in Figure 7. However, some parts of the walls are plastered by cement mortar, which damage the texture characteristics of the building's original surface, thus should be eliminated before renovation and protection design.

Xizhifang Village is a representative of dwellings in north Shandong (Figure 8). Using bricks and stones or sintered sun-dried mud as the foundation, the wall is mainly built by sun-dried mud made by mixing clay and straw. While the external layer of the wall is leveled by slurry. In renovation design of wall, high attention should be given for several parameters, such as size, proportion and building pattern of sun-dried mud bricks first,materials' quality and texture. The newly selected building materials should have the similar looseness, fineness or roughness with the original sun-dried mud brick. Besides, following the principle of "similar dominant colors" in visual perception, the newly added part should be in a color matching with yellowish-brown as the overall building is in yellowish brown. Additionally, it should also be noted that the coverage area should not go beyond that of the yellowish-brown part, or else, the dominant color will be changed.

Other than that, transparent building materials can be employed in renovation of dwellings in north Shandong. Such materials have their indistinct traits, thus having good match with the original building materials and remaining the architectural styles and characteristics of the original building, such as glass, PTFE, ETFE and other transparent composite materials. Among building materials, glass is the most common transparent building material. It featured with light weight, dense surface structure and easy operation and other advantages, thus it has been extensively applied in building renovation. ${ }^{[6]}$

In conclusion, increasingly much attention has been paid to the value of traditional dwellings as the new rural construction advances. The combination of old buildings and new functions are also attracting more architects' involvement. In turn, the protection and renovation of traditional dwellings has also arisen to be a hot topic for architects. ${ }^{[7]}$ This paper discusses the feasibility of dwellings in north Shandong by utilizing the above design methods. However, it should take note that it only serves as a small part in the study of traditional dwellings in north Shandong. Furthermore, in spite of arduous tasks in inheritance and development of traditional dwelling culture in north Shandong, it will be of far-reaching significance. ${ }^{[8]}$

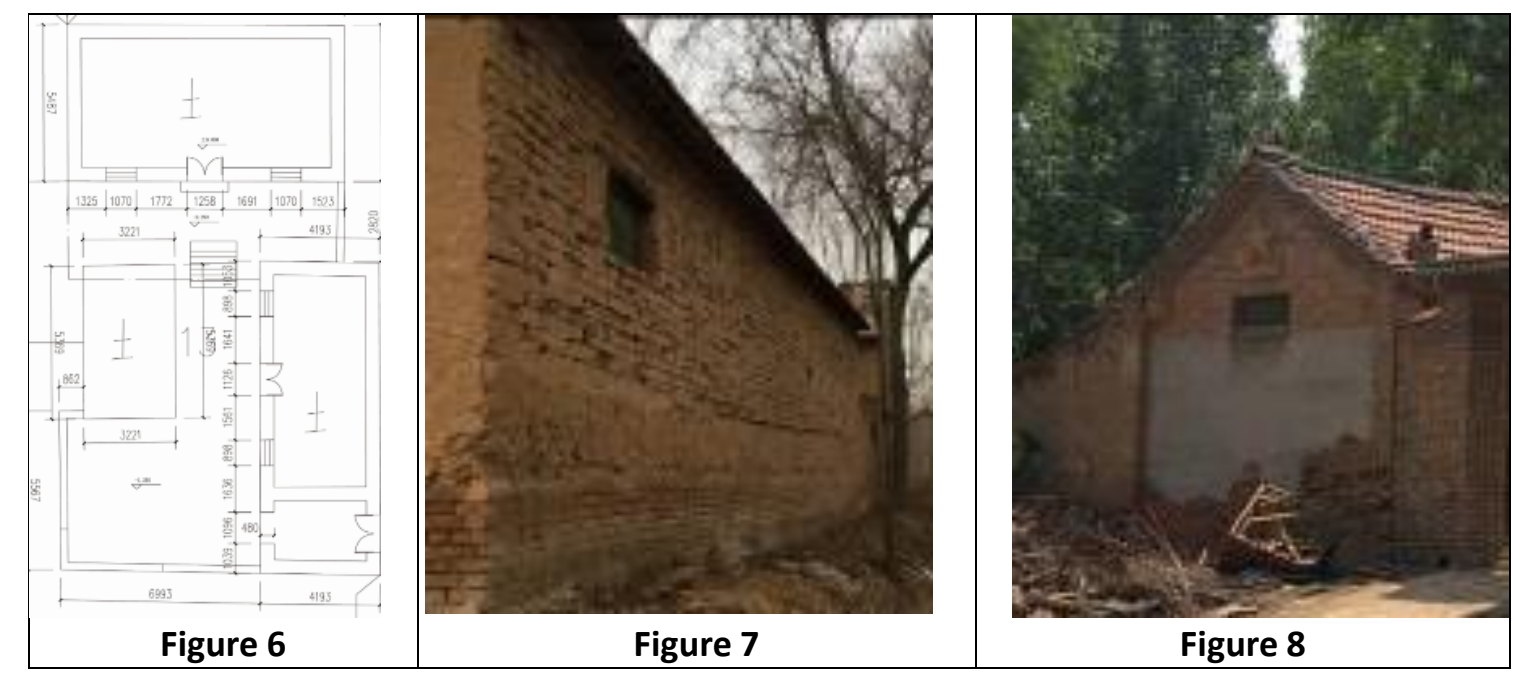

\section{References:}

[1] Xiaodan Duan, Building Renovation 2, China Architecture \& Building Press, 2002. (in Chinese)

36
[2] Study on "Design Methods for Concealed Renovation" of Existing Buildings, Yanru Gai, 2015, Master's Thesis. (in Chinese) 
[3] A Sauvegrain. Dialogues of Architectural Preservation in Modern Vietnam: The 36 Streets Commercial Quarter of Hanoi[J]. Traditional Dwellings \& Settlements Review, 2001,13(1):23-32.

[4] C Li, B Che. Study on the Protection and Development of Traditional Village Combined with the Folk Tourism: Case on Chelong Village in Meizhou City[J]. Architecture \& Culture, 2015. (in Chinese)

[5] TT Pham, DC He, D Morin. Relationship Between the Landscape Structure of Urban Green Spaces and Residents' Satisfaction: The Case of a Central District in Hanoi (Vietnam)[J]. Asian Journal of Geoinformatics, 2012.

[6] E Henry. Lauren Meeker, Sounding out Heritage: Cultural Politics and the Social Practice of Quan Ho Folk Song in North Vietnam[J]. Southeast Review of Asian Studies, 2014,36.

[7] PM Hoai, NT Ngoc, NH Minh, et al. Recent levels of organochlorine pesticides and polychlorinated biphenyls in sediments of the sewer system in Hanoi, Vietnam[J]. Environmental Pollution, 2010, 158(3): 913-920.

[8] TO Gods. Temples of the Earthbound GodsUniversity of Texas Press[J]. Geosphere, 2013, 9(3): 63-73. 